\title{
Sex differences in pain
}

\author{
Edmund Keogh \\ Senior Lecturer in Psychology, University of Bath
}

S U M M A RY P O I N T S

- There is good evidence that men and women differ with respect to the perception and experience of pain.

- Women on average report more pain when compared to men, and there seem to be more painful conditions where women exhibit a greater prevalence than where men do.

- Sex differences in pain vary according to age, with many differences occurring during the reproductive years.

- The sex of an individual is still not routinely investigated in many studies.

- There are a range of mechanisms that may be involved in explaining these sex differences, from sex hormones to health seeking behaviours.

\section{Introduction}

The past few years has seen the emergence of sex and gender as important issues in pain and analgesia ${ }^{1,2,3}$. Early investigations focused on whether there was any evidence for differences between the sexes. Questions have also been directed towards understanding why such differences exist, as well as to consider what should be done about them. The purpose of the current review is to present readers with a general overview of some of the research that has been conducted in this area, to hopefully demonstrate why many believe that sex and gender issues are important when thinking about pain and analgesia.

\section{Sex differences in pain perception}

Perhaps the best starting point when considering sex differences in pain is to examine whether there is any evidence that men and women perceive, experience and report pain in different ways. As will become apparent, such evidence does exist, stemming from a range of different sources, and suggests that on average women report more pain than men ${ }^{1,4,5}$. One area of investigation has been to examine the pain responses of healthy individuals using experimental pain induction procedures. The advantage of such procedures is that we can look at sex differences in laboratory environments, where measures of pain threshold and tolerance levels can be carefully controlled. Here there is evidence that there are sex differences in pain, with women usually exhibiting greater sensitivity (lower threshold, tolerance) to pain than men 6 . However, although these patterns are found across a range of different situations, it seems that some methods are associated with stronger sex effects than others. For example, in one meta-analysis of different types of experimental pain procedures, pressure pain and electrical stimulation were found to produce the strongest sex differences when compared to other methods ${ }^{6}$. Other factors were found to influence experimental pain variability, both between and within the sexes. For example, menstrual cycle phase is known to moderate sensitivity to experimental pain within women ${ }^{7}$. There are suggestions that for a number of pain induction methods, the luteal phase of the menstrual cycle is related to greater sensitivity to experimental pain. Interestingly, such cycle-related effects are not found within women on oral contraceptives, where there is a more stable hormonal profile, suggesting that sex hormones may play an important role in explaining the variation in pain sensitivity ${ }^{8}$. Other factors that are known to differentially impact on how males and females report pain in laboratory settings include psychosocial variables, such as emotions and social context. For example, it has been found that the sex of experimenter may influence whether male and female participants report pain; one study found that if the experimenter was female, then male participants reported less pain?. This suggests that psychosocial factors are important in how men and women respond to experimental pain, and that interpersonal interactions need to be considered.

\section{Sex differences in clinical pain and analgesic use}

The notion that there are sex differences in pain is not limited to the laboratory. Although healthy men and women show differences in pain within laboratory settings, such differences tend to be stronger when we consider clinical pain states ${ }^{2}$. In addition, there is evidence for sex-specific differences in the prevalence of some painful conditions. 
For example, fibromyalgia and rheumatoid arthritis show greater prevalence within women, whereas other conditions such as cluster headache tend to occur more in men. Interestingly, if these different painful conditions are compared, then we tend to see that there are more painful conditions where females show a greater prevalence, in comparison to the number of male-prevalent painful conditions ${ }^{1}$.

Epidemiological evidence also suggests that for a range of different painful conditions, women tend to report more pain and suffering than men. For example, in a larger review of published studies into sex differences in clinical pain, the general pattern was that women reported more severe, frequent and longer lasting pain than men ${ }^{5}$. The notion that men and women experience different pain has also been examined in the context of analgesic use; women report greater usage of both prescription and over the counter analgesics than men $\mathrm{do}^{10}$.

Although women seem to generally suffer from more pain than men, these differences are complicated by other factors, such as age $\mathrm{e}^{4}$. Sex differences in pain seem to depend on age, in that they tend to emerge during the reproductive years (i.e., puberty $\&$ menopause), with fewer observed differences found within children and older adults. Other factors that have been shown to influence variation in pain experience in a sex-specific manner include sex hormones, anxiety/depression, as well as interpersonal interactions. For example, pain anxiety may be more strongly related to pain in men, whereas the relationship between depression and pain may be stronger in women ${ }^{3}$. Also, one of the more consistent patterns that has emerged is the role that catastrophizing has in mediating sex differences in pain ${ }^{3}$. Thus as with experimental approaches, a wide range of biological, psychological and social influences are thought to impact on sex differences in clinical pain.

\section{Sex differences in responses to pain interventions}

If there are differences between the sexes in pain experiences and susceptibility to pain, then it is possible that there may be differences in how men and women respond to pain interventions. The experimental techniques reported above have been used to examine whether men and women respond in different ways to both pharmacological and non-pharmacological interventions. For example, sex differences in responses to analgesics have been examined using experimental paininduction methods ${ }^{11}$. Although there are relatively few studies, they suggest that sex differences exist, with some finding greater opioid analgesia in women than men. However, some of these effects may also depend on the type of pain induction method used. Alongside pharmacological interventions, there are also examples where psychological manipulations have been employed and responses to experimental pain measured. Again sex specific effects are reported. For example, we have found that asking healthy adults to employ different coping strategies has a differential effect on the pain experience of men and women ${ }^{12}$; focusing on pain was shown to be more beneficial to men when compared to women.

Alongside experimental studies on healthy volunteers there is evidence that men and women with clinical pain may differentially respond to interventions. For example, there may be differences between the sexes in terms of analgesic consumption. Indeed, one investigation found that women obtained greater analgesia following dental surgery than men $\mathrm{did}^{13}$. However, there are discrepancies, with some studies finding that female patients report more pain and required more morphine ${ }^{14}$. Reasons why there are differences in findings are complex, and so explanations are unclear at present. Another complication is that there may be sex differences in the side effects associated with drugs ${ }^{15}$. It is therefore possible that avoidance of unpleasant side effects could result in reduced demand for some analgesics, and that this may occur in a sex-specific manner.

Alongside sex differences in responses to pharmacological treatment for clinical pain, there is also evidence to suggest that differences may also exist for non-pharmacological interventions. For example, we have found evidence that men and women respond in different ways to an interdisciplinary 3 week pain management programme ${ }^{16}$. Although both male and female patients exhibited a benefit in terms of a range of outcomes immediately following the intervention, when assessed at a 3 month follow up point, sex differences were found. Specifically, women reported higher levels of pain and distress than men, even though both groups maintained improvements in disability at follow-up. Although other studies have also found sex differences in treatment outcomes for chronic pain, the results are mixed, with some reporting males benefit more, and others the opposite effect. Part of the problem is that there are too few studies that have explicitly examined sex differences in treatment outcome, and those that do vary considerably in terms of the intervention technique employed, thus preventing any general conclusions being made.

\section{Lack of research}

Clearly more research is required that focuses on isolating the situations and conditions that may be more likely to show (or not) differences in pain, both between and within the sexes. Part of the problem is that although many feel that sex and gender are important in pain, there are still too few investigations that explicitly design their studies to examine sex effects. Indeed, a review of 10 years of animal research published in the journal PAIN (1996-2005), found that 79\% of studies only included males, $8 \%$ only included females, and in $5 \%$, although both males and female were included, no report was made of any comparison between these groups; only $4 \%$ of studies explicitly examined for sex specific effects ${ }^{17}$. Such problems are not limited to pain studies, nor non human animal work. Indeed, there are issues for research with humans as well. For example, in a Health Technology Assessment review of inclusion within clinical trials conducted in the United Kingdom, the general conclusion was that the exclusion of women from clinical trials has not been adequately addressed ${ }^{18}$. Part of the problem could be due to a general lack of awareness of the key issues involved. In light of such concerns, a consensus document has recently been published that seeks to provide clearer guidance to those involved in pain research ${ }^{19}$, and part of IASP's 2007-2008 global campaign (which is against pain in women) seeks to raise general awareness about sex differences in pain. 


\section{Future directions}

What's next for sex/gender and pain? Perhaps the main issues to consider are why such differences exist, why there is variability within as well as between the sexes, and what the implications are for the management of pain. These questions are likely to be answered through further identification of the mechanisms that underpin such differences. As is apparent from the above review, explanations for sex differences are likely to be multifaceted, and reflect biological and psychosocial origins. Although it comes as no surprise to discover that the biopsychosocial approach is advocated as a useful way of conceptualising the many possible influences on pain, there are few studies that directly consider sex-gender interactions ${ }^{2}$. One possible reason for this could be that we are still some way off understanding the various influences and levels of analysis within these domains ${ }^{20}$.

Understanding why such sex differences occur could also have practical implications, in that it may help us decide on the most effective treatment approaches for men and women. Potential sex differences in the response and effectiveness of some analgesics could lead to different approaches. In terms of psychosocial interventions, we know that social support can be helpful, and that women tend to make greater use of this strategy than men $^{5}$. It may be the case that promoting social support may be useful for men. Similarly, given that catastrophizing is thought to account for some of the sex differences in pain, targeting this may be of particular use to women ${ }^{16}$. Such suggestions are of course purely speculative at present, and it is far too early to advocate any sex/gender specific intervention.

\section{Conclusions}

The evidence presented above suggests that there are potentially important differences between the sexes in pain perception and experience. There is also a suggestion that men and women differ with respect to how they respond to treatment interventions. Although this research suggests that there are important differences in pain experiences between the sexes, there is also considerable variability within the sexes that needs to be accounted for. Moreover, while sex may serve as an important risk factor with respect to pain, there are still too few studies that routinely examine for sex effects within a pain context. More research is required to understand when the sex of an individual may be important, as well as to further our understanding as to the mechanisms that may be involved. In doing so we should hopefully be in a better position to understand how best to manage pain in men and women.

\section{REFERENCES}

1. Berkley KJ. Sex differences in pain. Behav Brain Sci 1997; 20(3): 371-80.

2. Holdcroft A, Berkley KJ. Sex and gender differences in pain and its relief. In: McMahon SB, Koltzenburg M, Wall PD, Melzack R, editors. Wall and Melzack's textbook of pain. 5th ed. Edinburgh: Elsevier Churchill Livingstone 2005: p. 1181-97.

3. Keogh E. Sex and gender differences in pain: A selective review of biological and psychosocial factors. The Journal of Men's Health \& Gender 2006; 3(3): 236-43.

4. LeResche L. Gender considerations in the epidemiology of chronic pain. In: Crombie IK, Croft PR, Linton SJ, LeResche L, Von Korff M, editors. Epidemiology of pain. Seattle: IASP Press 1999: p. 43-52.

5. Unruh AM. Gender variations in clinical pain experience. Pain 1996; 65(2-3): 123-67.

6. Riley JL, Robinson ME, Wise EA, Myers CD, Fillingim RB. Sex differences in the perception of noxious experimental stimuli: A meta-analysis. Pain 1998; 74(2-3): 181-7.

7. Riley JL, Robinson ME, Wise EA, Price DD. A meta-analytic review of pain perception across the menstrual cycle. Pain 1999; 81(3): 225-35.

8. Craft RM, Mogil JS, Aloisi AM. Sex differences in pain and analgesia: The role of gonadal hormones. Eur J Pain 2004; 8(5): $397-411$.

9. Levine FM, Desimone LL. The effects of experimenter gender on pain report in male and female subjects. Pain 1991; 44(1): 6972.

10. Isacson D, Bingefors K. Epidemiology of analgesic use: A gender perspective. Eur J Anaesthesiol 2002; 19: 5-15.

11. Fillingim RB, Gear RW. Sex differences in opioid analgesia: Clinical and experimental findings. Eur J Pain 2004; 8(5): 41325.

12. Keogh E, Hatton K, Ellery D. Avoidance versus focused attention and the perception of pain: Differential effects for men and women. Pain 2000; 85(1-2): 225-30.

13. Gear RW, Miaskowski C, Gordon NC, Paul SM, Heller PH, Levine JD. Kappa-opioids produce significantly greater analgesia in women than in men. Nat Med 1996; 2(11): 1248-50.

14. Cepeda MS, Carr DB. Women experience more pain and require more morphine than men to achieve a similar degree of analgesia. Anesth Analg 2003; 97(5): 1464-8. 
15. Ciccone GK, Holdcroft A. Drugs and sex differences: A review of drugs relating to anaesthesia. BrJ Anaesth 1999; 82(2): 255-65.

16. Keogh E, McCracken LM, Eccleston C. Do men and women differ in their response to interdisciplinary chronic pain management? Pain 2005; 114(1-2): 37-46.

17. Mogil JS, Chanda ML. The case for the inclusion of female subjects in basic science studies of pain. Pain 2005; 117(1-2): 15.

18. Bartlett C, Doyal L, Ebrahim S, Davey P, Bachmann M, Egger M, Dieppe P. The causes and effects of socio-demographic exclusions from clinical trials. Health Technol Assess 2005; 9(38): 1-152.

19. Greenspan JD, Craft RM, LeResche L, Arendt-Nielsen L, Berkley KJ, Fillingim R, Gold M, Holdcroft A, Lautenbacher S, Mayer E. Studying sex and gender differences in pain and analgesia: A consensus report. Pain 2007; 132(Supplement 1): S26-S45.
20. Bernardes SF, Keogh E, Lima ML. Bridging the gap between pain and gender research: A selective literature review. Eur J Pain 2008; 12(4): 427-440.

CORRESPONDENCE TO:

Dr E Keogh, BSc, MSc, PhD

Senior Lecturer

Centre for Pain Research \& Department of Psychology,

University of Bath, Bath, BA2 7AY

Phone: 01225383671

Fax: $\quad 01225386752$

email: e.m.keogh@bath.ac.uk 